Research Paper

\title{
Association between sarcopenia and hearing thresholds in postmenopausal women
}

\author{
Seok Hui Kang ${ }^{1 *}$, Da Jung Jung ${ }^{2 *}$, Kyu Hyang Cho ${ }^{1}$, Jong Won Park ${ }^{1}$, Kyu-Yup Lee ${ }^{2}$, and Jun Young Do ${ }^{1 凶}$ \\ 1. Division of Nephrology, Department of Internal Medicine, Yeungnam University Hospital, Daegu, Republic of Korea; \\ 2. Department of Otorhinolaryngology-Head and Neck Surgery, School of Medicine, Kyungpook National University Hospital, Daegu, Republic of Korea. \\ * These authors contributed equally to this work. \\ $\square$ Corresponding author: Jun-Young Do, MD, Department of Internal Medicine, Yeungnam University Hospital, 317-1 Daemyung-Dong, Nam-Ku, Daegu \\ 705-717, Korea Fax: +82-53-654-8386, Phone: +82-53-680-3844, E-mail: jydo@med.yu.ac.kr \\ (c) Ivyspring International Publisher. This is an open access article distributed under the terms of the Creative Commons Attribution (CC BY-NC) license \\ (https://creativecommons.org/licenses/by-nc/4.0/). See http://ivyspring.com/terms for full terms and conditions.
}

Received: 2016.10.23; Accepted: 2017.01.31; Published: 2017.04.09

\begin{abstract}
Background: Given the association between metabolic disturbance and sarcopenia, sarcopenia may be intrinsically associated with the prevalence of HL. However, few studies describe the association between sarcopenia and $\mathrm{HL}$. The aim of this study was to evaluate the clinical association between sarcopenia and $\mathrm{HL}$ in postmenopausal Korean women.

Patients and Methods: A total of 4,038 women were ultimately included in this study. All participants were postmenopausal. Participants were divided into two groups based on criteria from the Foundation for the National Institute of Health Sarcopenia Project: a normal group (sarcopenia index $\geq 0.512$ ) and a sarcopenia group (sarcopenia index $<0.512$ ). Low-frequency (Low-Freq), mid-frequency (Mid-Freq), and high-frequency (High-Freq) values were obtained. The average hearing threshold (AHT) was calculated as the pure tone average at the 4 frequencies of $0.5 \mathrm{kHz}, 1 \mathrm{kHz}, 2 \mathrm{kHz}$, and $3 \mathrm{kHz}$. Mild HL was as an AHT of 24 to $40 \mathrm{~dB}$; moderate-to-profound $\mathrm{HL}$ was defined as an AHT of $40 \mathrm{~dB}$ or greater.

Results: Of the 4,038 participants, $272(6.7 \%)$ were allocated to the sarcopenia group, leaving $3,766(93.3 \%)$ in the normal group. The groups differed significantly in terms of having hypertension $(775[20.6 \%]$ vs. $108[39.7 \%] ; P<0.001)$ or metabolic syndrome $(817[21.7 \%]$ vs. 110 [40.4\%]; $P<0.001)$ in the normal and sarcopenia groups, respectively. Visceral fat area $\left(\mathrm{cm}^{3}\right)$ in the normal and sarcopenia groups was $99.0 \pm 21.9 \mathrm{~cm}^{3}$ and $117.0 \pm 21.8 \mathrm{~cm}^{3}$, respectively $(P<0.001)$. The hsCRP level was higher in the sarcopenia group than in the normal group. For univariate and multivariate analyses, all 4 hearing thresholds were higher in the sarcopenia group than in the normal group. In addition, linear regression analyses showed Low-Freq, Mid-Freq, and High-Freq to be inversely correlated with the sarcopenia index. The unadjusted OR for mild HL was 2.692 $(95 \% \mathrm{Cl}, 1.963-3.692 ; P<0.001)$ in the sarcopenia group relative to the normal group, with an adjusted $\mathrm{OR}$ of $1.584(95 \% \mathrm{Cl}, 1.131-2.217 ; P=0.007)$. The unadjusted $\mathrm{OR}$ for moderate-to-profound $\mathrm{HL}$ in the sarcopenia group relative to the normal group was 6.246 (95\% $\mathrm{Cl}, 4.530-8.612 ; P<0.001)$; the adjusted $\mathrm{OR}$ was $2.667(95 \% \mathrm{Cl}, 1.866-3.812 ; P<0.001)$.
\end{abstract}

Conclusion: Sarcopenia may be associated with HL. It may be beneficial to perform screening audiometry in patients with sarcopenia.

Key words: Sarcopenia, Hearing loss, Postmenopausal women, Hearing threshold.

\section{Background}

Sarcopenia, defined as decreased muscle mass, strength, and function, is a common pathologic or physiologic phenomenon in older people [1]. It can result in increased disability, hospitalization, comorbidity, and mortality. Initially, sarcopenia was defined as the loss of muscle mass associated with 
aging, but recent studies have shown an association between sarcopenia and various diseases [2-5]. Several factors, such as malnutrition, hormonal imbalance, and low vitamin D levels, are associated with the development of sarcopenia. In addition, recent studies have shown sarcopenia to be associated with metabolic disturbances such as atherosclerosis, insulin resistance, and dyslipidemia [6-8].

Hearing loss (HL) has a prevalence of approximately $4.9-17.0 \%$, making it a common public health problem. It is associated with decreased communication and decreased quality of life [9-11]. Aging, genetic factors, and use of ototoxic medications are well-known risk factors for HL. Recently, studies have also investigated the association between various metabolic disturbances and HL [12-15]. Metabolic disturbances are strongly related to cardiovascular disorders which are, in turn, associated with decreased blood supply to both cochlea and auditory nerve; this may result in the development of HL [16].

Given the association between metabolic disturbance and sarcopenia, sarcopenia may be intrinsically associated with the prevalence of HL. However, few studies describe the association between sarcopenia and HL. The aim of this study was to evaluate the clinical association between sarcopenia and HL in postmenopausal Korean women.

\section{Patients and Methods}

\section{Study population}

Data was collected from the medical records of 10,212 women who underwent voluntary routine health examinations at Yeungnam University Hospital between June 2008 and April 2014 (Figure 1). If patients underwent multiple examinations, data from the initial visit were used. Participants were excluded if aged younger than $50(n=4,842)$ or older than 80 years $(n=89)$, or if no data could be provided for sarcopenia or hearing thresholds $(\mathrm{n}=1,243)$. A total of 4,038 women were ultimately included in this study. All participants were postmenopausal. Ethical approval for this study was obtained from the institutional review board of Yeungnam University Hospital. The board waived the need for informed consent, as the subjects' records and information were anonymized and de-identified prior to analysis.

\section{Study variables}

Clinical and laboratory data collected during clinical examination included the following: age, serum creatinine $(\mathrm{mg} / \mathrm{dL})$, body mass index (BMI, $\left.\mathrm{kg} / \mathrm{m}^{2}\right)$, fasting blood glucose $(\mathrm{mg} / \mathrm{dL})$, total cholesterol (mg/dL), high-density lipoprotein (HDL) cholesterol levels (mg/dL), triglyceride levels $(\mathrm{mg} / \mathrm{dL})$, high sensitivity C-reactive protein levels (hsCRP, mg/dL), systolic blood pressure ( $\mathrm{mmHg}$ ), diastolic blood pressure $(\mathrm{mmHg})$, appendicular lean mass $(\mathrm{kg})$, visceral fat area $\left(\mathrm{cm}^{3}\right)$, and hearing thresholds.

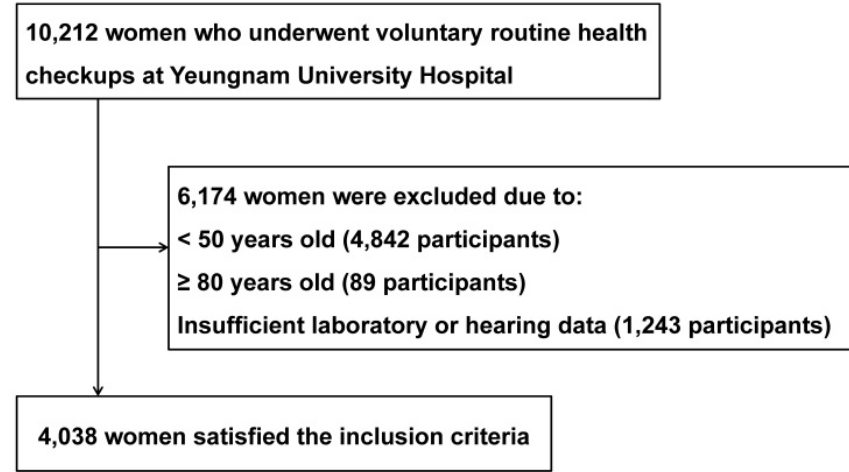

Figure 1. Study flow chart.

Appendicular lean mass and visceral fat area was measured using multi-frequency bioimepdance analysis (In-Body 720; Biospace, Seoul, Korea). Sarcopenia index was calculated as appendicular lean mass $(\mathrm{kg})$ divided by BMI $\left(\mathrm{kg} / \mathrm{m}^{2}\right)$. Participants were divided into two groups based on criteria from the Foundation for the National Institute of Health Sarcopenia Project: a normal group (sarcopenia index $\geq 0.512$ ) and a sarcopenia group (sarcopenia index < 0.512) [17]. Serum creatinine levels were measured using a Hitachi Automatic Analyzer (alkaline picrate, Jaffé kinetic). The estimated glomerular filtration rate (eGFR) was calculated using the Chronic Kidney Disease Epidemiology Collaboration (CKD-EPI) equation [18]. Chronic kidney disease was defined as an eGFR $<60 \mathrm{~mL} / \mathrm{min} / 1.73 \mathrm{~m}^{2}$. Diabetes mellitus (DM) was defined as a fasting blood glucose level $\geq$ $126 \mathrm{mg} / \mathrm{dL}$ or a self-reported history of DM. Hypertension was defined as a systolic blood pressure measurement of $\geq 140 \mathrm{mmHg}$ or a diastolic measurement of $\geq 90 \mathrm{mmHg}$ or a self-reported history of hypertension. The National Cholesterol Education Program Adult Treatment Panel III guidelines were used to define the metabolic syndrome [18].

Hearing thresholds were measured using an automatic audiometer at $0.5 \mathrm{kHz}, 1 \mathrm{kHz}, 2 \mathrm{kHz}, 3$ $\mathrm{kHz}, 4 \mathrm{kHz}$, and $6 \mathrm{kHz}$. None of the participants had been receiving ototoxic medication and none had ear disease (e.g., chronic otitis media). Hearing was tested in both ears of each participant. Low-frequency (Low-Freq), mid-frequency (Mid-Freq), and high-frequency (High-Freq) values were obtained calculating the pure tone averages at 0.5 and $1 \mathrm{kHz}, 2$ and $3 \mathrm{kHz}$, and 4 and $6 \mathrm{kHz}$, respectively. The average 
hearing threshold (AHT) was calculated as the pure tone average at the 4 frequencies of $0.5 \mathrm{kHz}, 1 \mathrm{kHz}, 2$ $\mathrm{kHz}$, and $3 \mathrm{kHz}$. Mild HL was as an AHT of 24 to 40 $\mathrm{dB}$; moderate-to-profound HL was defined as an AHT of $40 \mathrm{~dB}$ or greater.

\section{Statistical analyses}

The data were analyzed using the Statistical package for the Social Sciences software package (SPSS v.21, Chicago, IL., USA). Categorical variables were expressed as both counts and percentages. Continuous variables were expressed as the mean \pm standard deviation (SD) or standard error (SE). The Pearson's $\chi^{2}$ or Fisher's exact test was used to analyze categorical variables, as appropriate. For continuous variables, Student's t-test was used to compare the means.

Linear regression analysis was performed to assess independent predictors of hearing thresholds. Logistic regression analyses were used to estimate odds ratios (OR) and 95\% confidence intervals (CI), which were then used to determine the association between sarcopenia and HL.

The multivariate analysis was adjusted for age, diabetes mellitus, hypertension, eGFR, total cholesterol, HDL cholesterol, and triglyceride level. Multivariate analyses using analyses of covariance, multiple linear regression, or multiple logistic regression were used determine the independent predictors of HL. Discrimination- which is the ability of the model to differentiate between participants who have HL and those who do not -was examined using the area under the receiver operating characteristic (AUROC) curve. AUROC analysis was also performed in order to calculate sensitivity and specificity. The AUROC was calculated using the MedCalc software package (v.11.6.1.0, MedCalc, Mariakerke, Belgium). A $P$-value $<0.05$ was considered statistically significant.

\section{Results}

\section{Clinical characteristics of participants}

Of the 4,038 participants, 272 (6.7\%) were allocated to the sarcopenia group, leaving 3,766 $(93.3 \%)$ in the normal group (Table 1). Sarcopenia index in the normal and sarcopenia groups was 0.656 \pm 0.080 and $0.479 \pm 0.030$, respectively. Age, BMI, fasting blood glucose, total cholesterol, triglyceride levels, and systolic and diastolic blood pressure were higher in the sarcopenia group than in the normal group. HDL cholesterol level and eGFR were lower in the sarcopenia group than in the normal group. In the normal group, 396 (10.5\%) participants had DM, vs. 51 $(18.8 \%)$ in the sarcopenia group $(P<0.001)$. The groups also differed significantly in terms of having hypertension (775 [20.6\%] vs. 108 [39.7\%]; $P<0.001$ ) or metabolic syndrome (817 [21.7\%] vs. 110 [40.4\%]; $P$ $<0.001$ ) in the normal and sarcopenia groups, respectively. The number of metabolic syndrome components in the normal and sarcopenia groups was $1.62 \pm 1.12$ and $2.23 \pm 0.98$, respectively $(P<0.001)$. Visceral fat area $\left(\mathrm{cm}^{3}\right)$ in the normal and sarcopenia groups was $99.0 \pm 21.9 \mathrm{~cm}^{3}$ and $117.0 \pm 21.8 \mathrm{~cm}^{3}$, respectively $(P<0.001)$. The hsCRP level was higher in the sarcopenia group than in the normal group.

Table 1. Clinical characteristics of participants

\begin{tabular}{llll}
\hline & $\begin{array}{l}\text { Normal } \\
\text { (n= 3,766) }\end{array}$ & $\begin{array}{l}\text { Sarcopenia } \\
\text { (n= 272) }\end{array}$ & P-value $^{*}$ \\
\hline Age (years) & $59.5 \pm 7.4$ & $67.7 \pm 7.6$ & $<0.001$ \\
Body mass index $\left(\mathrm{kg} / \mathrm{m}^{2}\right)$ & $23.8 \pm 2.8$ & $26.7 \pm 3.8$ & $<0.001$ \\
Fasting blood glucose $(\mathrm{mg} / \mathrm{dL})$ & $95.2 \pm 21.6$ & $100.4 \pm 26.0$ & $<0.001$ \\
Systolic blood pressure $(\mathrm{mmHg})$ & $120 \pm 15$ & $128 \pm 16$ & $<0.001$ \\
Diastolic blood pressure (mmHg) & $76 \pm 11$ & $80 \pm 11$ & $<0.001$ \\
eGFR(mL/min/1.73 m²) & $91.2 \pm 26.0$ & $87.6 \pm 19.7$ & 0.027 \\
Total cholesterol (mg/dL) & $208.0 \pm 37.8$ & $213.9 \pm 39.6$ & 0.013 \\
Triglyceride (mg/dL) & $116.7 \pm 76.9$ & $135.4 \pm 71.9$ & 0.010 \\
HDL cholesterol (mg/dL) & $59.2 \pm 15.0$ & $56.7 \pm 15.1$ & $<0.001$ \\
hsCRP (mg/dL) & $0.16 \pm 0.55$ & $0.31 \pm 1.22$ & $<0.001$ \\
Sarcopenia index & $0.656 \pm 0.080$ & $0.479 \pm 0.030$ & $<0.001$ \\
\hline
\end{tabular}

Data are expressed as numbers (percentages) for categorical variables and mean \pm standard deviations for continuous variables.

${ }^{*} P$ values were tested by student $\mathrm{t}$-test. Abbreviations: eGFR, estimated glomerular filtration rate; HDL, high-density lipoprotein; hsCRP, high sensitivity C-reactive protein.

\section{Association between sarcopenia and hearing thresholds}

Hearing thresholds (mean \pm SE) in the normal and sarcopenia groups were $25.6 \pm 0.2 \mathrm{~dB}$ and $34.6 \pm$ $1.0 \mathrm{~dB}$ in Low-Freq, $28.8 \pm 0.2 \mathrm{~dB}$ and $41.1 \pm 1.2 \mathrm{~dB}$ in Mid-Freq, $35.2 \pm 0.3 \mathrm{~dB}$ and $51.6 \pm 1.2 \mathrm{~dB}$ in High-Freq, and $27.2 \pm 0.2 \mathrm{~dB}$ and $37.9 \pm 1.0 \mathrm{~dB}$ in AHT (Figure 2). Multivariate analysis revealed that hearing thresholds in the normal and sarcopenia groups were $25.7 \pm 0.2$ $\mathrm{dB}$ and $33.7 \pm 0.7 \mathrm{~dB}$ in Low-Freq, $28.9 \pm 0.2 \mathrm{~dB}$ and $39.8 \pm 0.9 \mathrm{~dB}$ in Mid-Freq, $35.4 \pm 0.3 \mathrm{~dB}$ and $49.8 \pm 1.1$ $\mathrm{dB}$ in High-Freq, and $27.3 \pm 0.2 \mathrm{~dB}$ and $36.8 \pm 0.8 \mathrm{~dB}$ in AHT. For univariate and multivariate analyses, all 4 hearing thresholds were higher in the sarcopenia group than in the normal group. In addition, linear regression analyses showed Low-Freq, Mid-Freq, and High-Freq to be inversely correlated with the sarcopenia index (Table 2). 


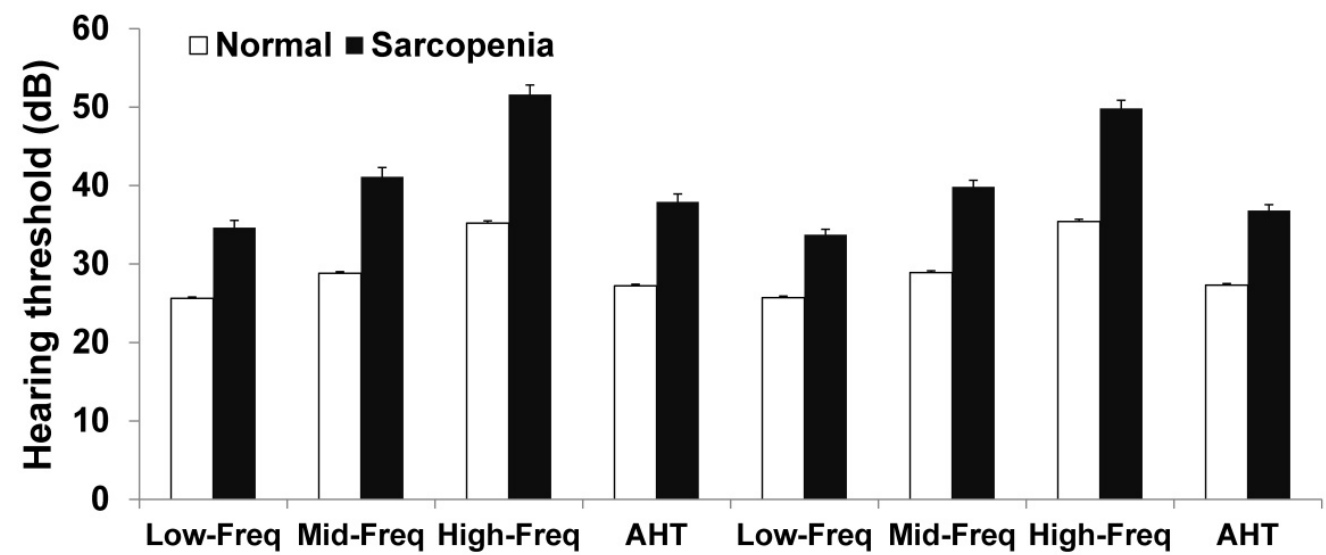

\section{Univariate}

Multivariate

Figure 2. Hearing thresholds according to the presence of sarcopenia. The multivariate analysis was adjusted for age, diabetes mellitus, hypertension, estimated glomerular filtration rate, total cholesterol level, high-density lipoprotein level, and triglyceride level $(P<0.001$ for trend in all analyses). The data are expressed as mean and standard error values. Abbreviation: Low-Freq, low frequency; Mid-Freq, middle frequency; High-Freq, high frequency; AHT, average hearing threshold.

Table 2. Linear regression analyses of variable hearing thresholds by sarcopenia index.

\begin{tabular}{|c|c|c|c|c|}
\hline & \multicolumn{2}{|l|}{ Univariate } & \multicolumn{2}{|l|}{ Multivariate } \\
\hline & Standardized $\beta \pm \mathrm{SE}$ & $P$-value* & Standardized $\beta \pm \mathrm{SE}$ & $P$-value* \\
\hline \multicolumn{5}{|l|}{ Dep: Low-Freq } \\
\hline Sarcopenia index & $--0.257 \pm 2.005$ & $<0.001$ & $-0.067 \pm 2.059$ & $<0.001$ \\
\hline Age & $0.462 \pm 0.021$ & $<0.001$ & $0.441 \pm 0.025$ & $<0.001$ \\
\hline Fasting blood glucose & $0.107 \pm 0.008$ & $<0.001$ & $0.038 \pm 0.008$ & 0.008 \\
\hline Systolic blood pressure & $0.133 \pm 0.012$ & $<0.001$ & $-0.074 \pm 0.023$ & 0.015 \\
\hline Diastolic blood pressure & $0.119 \pm 0.017$ & $<0.001$ & $0.060 \pm 0.032$ & 0.046 \\
\hline eGFR & $-0.058 \pm 0.007$ & $<0.001$ & $0.045 \pm 0.007$ & 0.002 \\
\hline Total cholesterol & $-0.019 \pm 0.005$ & 0.227 & - & - \\
\hline HDL cholesterol & $-0.103 \pm 0.012$ & $<0.001$ & $-0.044 \pm 0.012$ & 0.003 \\
\hline Triglyceride & $0.082 \pm 0.002$ & $<0.001$ & $-0.004 \pm 0.002$ & 0.786 \\
\hline \multicolumn{5}{|l|}{ Dep: Mid-Freq } \\
\hline Sarcopenia index & $-0.286 \pm 2.462$ & $<0.001$ & $-0.079 \pm 2.747$ & $<0.001$ \\
\hline Age & $0.506 \pm 0.026$ & $<0.001$ & $0.478 \pm 0.030$ & $<0.001$ \\
\hline Fasting blood glucose & $0.114 \pm 0.010$ & $<0.001$ & $0.038 \pm 0.009$ & 0.007 \\
\hline Systolic blood pressure & $0.151 \pm 0.014$ & $<0.001$ & $-0.079 \pm 0.028$ & 0.008 \\
\hline Diastolic blood pressure & $0.136 \pm 0.021$ & $<0.001$ & $0.070 \pm 0.039$ & 0.016 \\
\hline eGFR & $-0.073 \pm 0.009$ & $<0.001$ & $0.038 \pm 0.008$ & 0.006 \\
\hline Total cholesterol & $-0.013 \pm 0.006$ & 0.399 & - & - \\
\hline HDL cholesterol & $-0.118 \pm 0.015$ & $<0.001$ & $-0.055 \pm 0.014$ & $<0.001$ \\
\hline Triglyceride & $0.088 \pm 0.003$ & $<0.001$ & $-0.010 \pm 0.003$ & 0.050 \\
\hline \multicolumn{5}{|l|}{ Dep: High-Freq } \\
\hline Sarcopenia index & $-0.327 \pm 2.999$ & $<0.001$ & $-0.083 \pm 2.847$ & $<0.001$ \\
\hline Age & $0.593 \pm 0.030$ & $<0.001$ & $0.557 \pm 0.035$ & $<0.001$ \\
\hline Fasting blood glucose & $0.136 \pm 0.013$ & $<0.001$ & $0.045 \pm 0.011$ & 0.001 \\
\hline Systolic blood pressure & $0.180 \pm 0.018$ & $<0.001$ & $-0.044 \pm 0.032$ & 0.115 \\
\hline Diastolic blood pressure & $0.152 \pm 0.026$ & $<0.001$ & $0.034 \pm 0.045$ & 0.214 \\
\hline eGFR & $-0.102 \pm 0.011$ & $<0.001$ & $0.029 \pm 0.009$ & 0.025 \\
\hline Total cholesterol & $-0.011 \pm 0.007$ & 0.467 & - & - \\
\hline HDL cholesterol & $-0.133 \pm 0.019$ & $<0.001$ & $-0.055 \pm 0.016$ & $<0.001$ \\
\hline Triglyceride & $0.108 \pm 0.004$ & $<0.001$ & $-0.002 \pm 0.003$ & 0.871 \\
\hline
\end{tabular}

${ }^{*}$ The dependent variable was Low-Freq, Mid-Freq, or High-Freq, and independent variables were sarcopenia index, age, fasting blood glucose level, systolic blood pressure, diastolic blood pressure, eGFR, total cholesterol level, HDL cholesterol level, and triglyceride level.

Multivariate analysis was adjusted for sarcopenia index, age, fasting blood glucose level, systolic blood pressure, diastolic blood pressure, eGFR, HDL cholesterol level, and triglyceride level.

Abbreviations: Low-Freq, low frequency; Mid-Freq, mid-frequency; High-Freq, high-frequency; eGFR, estimated glomerular filtration rate; HDL, high-density lipoprotein. 


\section{Association between sarcopenia and hearing loss}

The AUROCs of the sarcopenia index for mild and moderate-to-profound HL were 0.630 (95\% CI, $0.615-0.645)$ and 0.672 (95\% CI, 0.657-0.686), respectively (Figure 3). The sensitivity and specificity for predicting HL were $53.2 \%$ and $65.9 \%$ in mild HL and $55.7 \%$ and $69.4 \%$ in moderate-to-profound HL, respectively.

The prevalence of mild or moderate-to-profound HL was $30.7 \%$ and $13.5 \%$ in the normal group, and $37.1 \%$ and $37.9 \%$ in the sarcopenia group. The prevalence of HL was higher in the sarcopenia group than in the normal group $(P<0.001)$. The unadjusted OR for mild HL was 2.692 (95\% CI, 1.963-3.692; $P<$ 0.001 ) in the sarcopenia group relative to the normal group, with an adjusted OR of $1.584(95 \% \mathrm{CI}$, 1.131-2.217; $P=0.007)$. The unadjusted $\mathrm{OR}$ for moderate-to-profound $\mathrm{HL}$ in the sarcopenia group relative to the normal group was 6.246 (95\% CI, 4.530-8.612; $P<0.001)$; the adjusted OR was 2.667 (95\% CI, 1.866-3.812; $P<0.001)$.

\section{Subgroup analysis by age}

For participants aged 50-59 years and 70-79 years, all 4 hearing thresholds were higher in the sarcopenia group than in the normal group. For those aged 60-69 years, there was a significant difference only in the High-Freq hearing threshold between the two groups (Table 3 ). The univariate and multivariate analyses showed similar results.
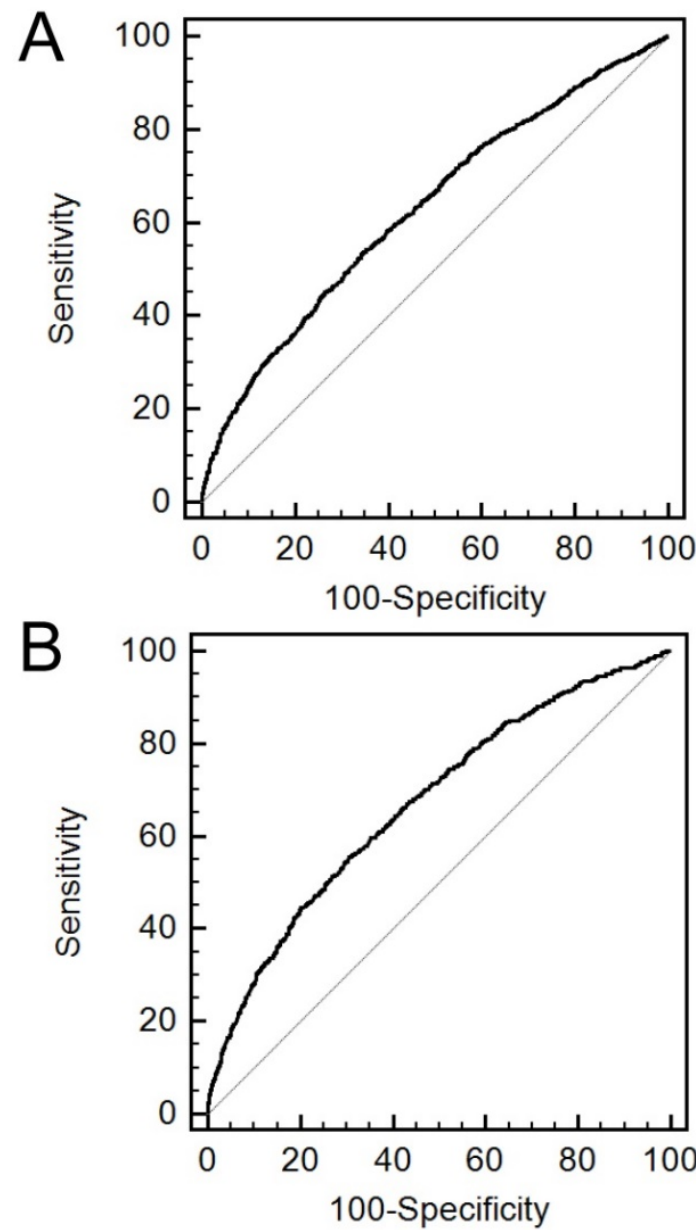

Figure 3. Receiver operating characteristic curves of sarcopenia index for the prediction of hearing loss. A. mild hearing loss. B. moderate-to-profound hearing loss.

Table 3. Subgroup analyses for the association between hearing thresholds and sarcopenia

\begin{tabular}{|c|c|c|c|c|c|c|}
\hline & \multicolumn{3}{|l|}{ Univariate } & \multicolumn{3}{|c|}{ Multivariate } \\
\hline & Normal & Sarcopenia & $P$-value* & Normal & Sarcopenia & $P$-value* \\
\hline \multicolumn{7}{|l|}{$50-59$ aged } \\
\hline Low-Freq & $22.1 \pm 0.2$ & $23.9 \pm 0.2$ & $<0.001$ & $22.1 \pm 8.2$ & $26.7 \pm 1.2$ & 0.002 \\
\hline Mid-Freq & $23.9 \pm 0.2$ & $22.0 \pm 3.3$ & $<0.001$ & $23.9 \pm 10.2$ & $32.6 \pm 1.6$ & $<0.001$ \\
\hline High-Freq & $28.0 \pm 0.3$ & $36.6 \pm 2.6$ & $<0.001$ & $28.0 \pm 12.9$ & $36.6 \pm 1.6$ & $<0.001$ \\
\hline $\mathrm{AHT}$ & $23.0 \pm 0.2$ & $29.7 \pm 2.1$ & $<0.001$ & $23.0 \pm 8.7$ & $28.7 \pm 1.3$ & $<0.001$ \\
\hline \multicolumn{7}{|l|}{$60-69$ aged } \\
\hline Low-Freq & $28.1 \pm 0.3$ & $30.5 \pm 1.4$ & 0.051 & $28.0 \pm 0.3$ & $30.0 \pm 1.2$ & 0.105 \\
\hline Mid-Freq & $32.4 \pm 0.4$ & $35.0 \pm 1.7$ & 0.078 & $32.3 \pm 0.4$ & $34.2 \pm 1.4$ & 0.197 \\
\hline High-Freq & $40.8 \pm 0.5$ & $46.2 \pm 1.9$ & 0.003 & $41.0 \pm 0.5$ & $44.7 \pm 1.7$ & 0.031 \\
\hline AHT & $30.2 \pm 0.4$ & $32.8 \pm 1.5$ & 0.055 & $30.2 \pm 0.4$ & $32.1 \pm 1.2$ & 0.133 \\
\hline \multicolumn{7}{|l|}{$70-79$ aged } \\
\hline Low-Freq & $35.6 \pm 0.6$ & $40.3 \pm 1.5$ & 0.001 & $35.7 \pm 0.7$ & $39.5 \pm 1.3$ & 0.009 \\
\hline Mid-Freq & $42.2 \pm 0.7$ & $48.4 \pm 1.6$ & $<0.001$ & $42.4 \pm 0.7$ & $47.6 \pm 1.4$ & 0.002 \\
\hline High-Freq & $54.3 \pm 0.8$ & $60.7 \pm 1.5$ & $<0.001$ & $54.6 \pm 0.8$ & $59.8 \pm 1.5$ & 0.002 \\
\hline AHT & $38.9 \pm 0.6$ & $44.3 \pm 1.5$ & $<0.001$ & $39.1 \pm 0.7$ & $43.5 \pm 1.3$ & 0.003 \\
\hline
\end{tabular}

Data are expressed as mean \pm standard errors.

${ }^{*} P$ values were tested by student $\mathrm{t}$-test for univariate analysis and by analysis of covariance for multivariate analysis. Multivariate analysis was adjusted for sarcopenia index, age, fasting blood glucose level, systolic blood pressure, diastolic blood pressure, eGFR, HDL cholesterol level, and triglyceride level.

Abbreviations: Low-Freq, low frequency; Mid-Freq, mid-frequency; High-Freq, high-frequency; eGFR, estimated glomerular filtration rate; HDL, high-density lipoprotein. 


\section{Discussion}

Our study included only postmenopausal women for exclusion of sex or hormonal effects. Participants were divided into a sarcopenia and normal group based on the sarcopenia index. The sarcopenia and normal groups were compared with respect to hearing impairment and HL. Participants in the sarcopenia were significantly older and a greater proportion met the criteria for the metabolic syndrome than those without sarcopenia. The sarcopenia index had a significant AUC for prediction of mild or moderate-to-profound $\mathrm{HL}$ and was inversely correlated with hearing thresholds. All 4 hearing thresholds were higher in the participants with sarcopenia than in those without sarcopenia. In addition, logistic regression analyses showed an association between the presence of sarcopenia and mild or moderate-to-profound HL. These results indicate that sarcopenia may be associated with hearing impairment through the mechanism of increased metabolic disturbance in postmenopausal women.

The association between sarcopenia and metabolic disturbance is well-known, but the association is very complex. Sarcopenia can cause metabolic disturbances; conversely, metabolic disturbances can induce the sarcopenia. Muscles utilize large amounts of glucose, and decreases in muscle bulk can increase the risk of insulin resistance $[20,21]$. Metabolic syndrome is associated with increased visceral fat mass. Such an increase is associated with inadequate activation of the PI3/Akt signaling pathway by means of insulin resistance, and resulting in accelerated breakdown of muscle mass [22]. Chronic inflammation caused by the metabolic syndrome can also play a role in decreasing muscle mass. Our results demonstrate that sarcopenia is associated with both the metabolic syndrome and chronic inflammation. These factors can lead to endothelial damage in stria vascularis, which can result in hearing impairment $[23,24]$.

Our results show an association between sarcopenia and hearing thresholds (as a continuous variable) and $\mathrm{HL}$ (as a categorical variable). The predictive effect in moderate-to-profound HL was higher than that in mild HL. We postulate that this association is a result of metabolic disturbances combined with sarcopenia. Although we did not evaluate causality between sarcopenia and metabolic disturbances, these results indicate that participants with sarcopenia should be closely monitored for hearing impairment.

Age is an important confounding factor as it is associated with both sarcopenia and hearing impairment. Thus, we adjusted for age in the multivariate analysis. In addition, we tried to decrease the confounding effect of age by stratification into three subgroups (50-59; 50-69 and 70-79 years of age). In the subgroup analysis, Low-, Mid-, High-Freq, and AHT were significantly higher in the sarcopenia group than in the normal group amongst those aged 50-59 and 70-79. Amongst participants aged 60-69, only the High-Freq hearing threshold was significantly higher in the sarcopenia group than in the normal group. Although no statistically significance observed, the other hearing thresholds in the sarcopenia group showed a higher trend than in those in the normal group.

This study has a number of limitations. First, as it is a retrospective cross-sectional design, it cannot establish causality between sarcopenia and metabolic disturbances or between sarcopenia and hearing impairment or HL. Second, the study included an ethnically homogenous population at a single center, affecting generalizability. Third, this study did not evaluate sensitive components of either hearing impairment (such as speech discrimination) or sarcopenia (such as muscle strength).

In conclusion, sarcopenia may be associated with HL. Further prospective studies are needed to evaluate the strength of the association between sarcopenia and HL. However, it may be beneficial to perform screening audiometry in patients with sarcopenia.

\section{Acknowledgement}

This work was supported by the Medical Research Center Program (2015R1A5A2009124) through the National Research Foundation of Korea (NRF) funded by the Ministry of Science, ICT and Future Planning.

\section{Competing Interests}

The authors have declared that no competing interest exists.

\section{References}

[1] Studenski SA, Peters KW, Alley DE, Cawthon PM, McLean RR, Harris TB, et al. The FNIH sarcopenia project: rationale, study description, conference recommendations, and final estimates. J Gerontol A Biol Sci Med Sci. 2014;69:547-58.

[2] Foley RN, Wang C, Ishani A, Collins AJ, Murray AM. Kidney function and sarcopenia in the United States general population: NHANES III. Am J Nephrol. 2007;27:279-86.

[3] Morley JE, Vellas B, van Kan GA, Anker SD, Bauer JM, Bernabei R, et al. Frailty consensus: a call to action. J Am Med Dir Assoc. 2013;14:392-97.

[4] Cruz-Jentoft AJ, Baeyens JP, Bauer JM, Boirie Y, Cederholm T, Landi F, et al. European Working Group on Sarcopenia in Older People. Sarcopenia: European consensus on definition and diagnosis: Report of the European Working Group on Sarcopenia in Older People. Age Ageing. 2010;39:412-3.

[5] Buford TW, Anton SD, Judge AR, Marzetti E, Wohlgemuth SE, Carter CS, et al. Models of accelerated sarcopenia: critical pieces for solving the puzzle of age-related muscle atrophy. Ageing Res Rev. 2010;9:369-83.

[6] Dominguez LJ, Barbagallo M. The cardiometabolic syndrome and sarcopenic obesity in older persons. J Cardiometab Syndr. 2007;2:183-9. 
[7] Lee SW, Youm Y, Lee WJ, Choi W, Chu SH, Park YR, et al. Appendicular skeletal muscle mass and insulin resistance in an elderly Korean population: the Korean Social Life, Health and Aging Project-Health Examination cohort. Diabetes Metab J. 2015;39:37-45.

[8] Kim BC, Kim MK, Han K, Lee SY, Ko SH, Kwon HS, et al. Low muscle mass is associated with metabolic syndrome only in nonobese young adults: the Korea National Health and Nutrition Examination Survey 2008-2010. Nutr Res. 2015;35:1070-8.

[9] Jennings MB, Shaw L. Impact of hearing loss in the workplace: raising questions about partnerships with professionals. Work. 2008;30:289-95.

[10] Walling AD, Dickson GM. Hearing loss in older adults. Am Fam Physician. 2012;85:1150-6.

[11] Stevens G, Flaxman S, Brunskill E, Mascarenhas M, Mathers CD, Finucane M, et al. Global and regional hearing impairment prevalence: an analysis of 42 studies in 29 countries. Eur J Public Health. 2013;23:146-52.

[12] Agrawal Y, Platz EA, Niparko JK. Risk factors for hearing loss in US adults: data from the National Health and Nutrition Examination Survey, 1999 to 2002. Otol Neurotol. 2009;30:139-45.

[13] Van Eyken E, Van Camp G, Van Laer L. The complexity of age-related hearing impairment: contributing environmental and genetic factors. Audiol Neurootol. 2007;12:345-58.

[14] Kang SH, Jung da J, Cho KH, Park JW, Yoon KW, Do JY. The association between metabolic syndrome or chronic kidney disease and hearing thresholds in Koreans: the Korean National Health and Nutrition Examination Survey 2009-2012. PLoS One. 2015;10:e120372.

[15] Sun YS, Fang WH, Kao TW, Yang HF, Peng TC, Wu LW, et al. Component of metabolic syndrome as risk factors for hearing thresholds shifts. PLoS One. 2015;10:e0134388

[16] Oron Y, Elgart K, Marom T, Roth Y. Cardiovascular risk factors as causes for hearing impairment. Audiol Neurootol. 2014;19:256-60.

[17] Studenski SA, Peters KW, Alley DE, Cawthon PM, McLean RR, Harris TB, et al. The FNIH sarcopenia project: rationale, study description, conference recommendations, and final estimates. J Gerontol A Biol Sci Med Sci. 2014;69:547-58.

[18] Levey AS, Stevens LA, Schmid CH, Zhang YL, Castro AF 3rd, Feldman HI, et al. A new equation to estimate glomerular filtration rate. Ann Intern Med. 2009;150:604-12.

[19] Grundy SM, Cleeman JI, Daniels SR, Donato KA, Eckel RH, Franklin BA, et al. Diagnostic and management of the metabolic syndrome: an American Heart Association/National Heart, Lung, and Blood Institute Scientific Statement. Circulation. 2005;112:2735-52.

[20] Karlsson HK, Zierath JR. Insulin signaling and glucose transport in insulin resistant human skeletal muscle. Cell Biochem Biophys. 2007;48:103-13.

[21] Srikanthan P, Karlamangla AS. Relative muscle mass is inversely associated with insulin resistance and prediabetes. Findings from the third National Health and Nutrition Examination Survey. J Clin Endocrinol Metab. 2011;96:2898-903.

[22] Wang $\mathrm{X}, \mathrm{Hu} \mathrm{Z}, \mathrm{Hu} \mathrm{J}, \mathrm{Du} J$, Mitch WE. Insulin resistance accelerates muscle protein degradation: Activation of the ubiquitin-proteasome pathway by defects in muscle cell signaling. Endocrinology. 2006;147:4160-8.

[23] Fetoni AR, Picciotti PM, Paludetti G, Troiani D. Pathogenesis of presbycusis in animal models: a review. Exp Gerontol. 2011;46:413-425.

[24] Yamasoba T, Lin FR, Someya S, Kashio A, Sakamoto T, Kondo K. Current concepts in age-related hearing loss: epidemiology and mechanistic pathways. Hear Res. 2013;303:30-38. 